\title{
OPEN Distinct microbiome profiles and biofilms in Leishmania donovani-driven cutaneous leishmaniasis wounds
}

\author{
T. D. Jayasena Kaluarachchi ${ }^{1 凶}$, Paul M. Campbell ${ }^{2}$, Rajitha Wickremasinghe ${ }^{3}$, \\ Shalindra Ranasinghe ${ }^{1}$, Renu Wickremasinghe ${ }^{1}$, Surangi Yasawardene ${ }^{4}$, Hiromel De Silva ${ }^{5}$, \\ Chandrani Menike ${ }^{1}$, M. C. K. Jayarathne ${ }^{6}$, Subodha Jayathilake ${ }^{7}$ Ayomi Dilhari $^{8}$, \\ Andrew J. McBain ${ }^{2} \&$ Manjula M. Weerasekera ${ }^{9}$
}

The endemic strain of Leishmania donovani in Sri Lanka causes cutaneous leishmaniasis (CL) rather than more common visceral form. We have visualized biofilms and profiled the microbiome of lesions and unaffected skin in thirty-nine CL patients. Twenty-four lesions (61.5\%) were biofilm-positive according to fluorescence in situ hybridization. Biopsies of biofilm-positive lesions were dominated by Pseudomonas, class Bacilli and Enterobacteriaceae and distinguished by significantly lower community evenness. Higher relative abundance of a class BacilliOTU was detected in wound swabs versus contralateral skin. Wound swabs and biopsies had significantly distinct microbiome profiles and lower diversity compared to unaffected skin. Greater abundances of potentially pathogenic organisms were observed in wet ulcers, lesions with high parasite loads and large wounds. In summary, more than half of $L$. donovani associated $C L$ wounds harboured biofilms and the wounds exhibited a distinct, less diverse, microbiome than unaffected skin.

Leishmania donovani-driven cutaneous leishmaniasis is endemic to Sri Lanka. L. donovani, which usually causes visceral leishmaniasis, causes cutaneous leishmaniasis in Sri Lanka, parts of India, Sudan, Lebanon, and Turkey ${ }^{1}$. Cutaneous leishmaniasis can lead to disfiguring ulcers, and the lesions are chronic, taking months to years to completely heal (with or without anti-parasitic treatment) ${ }^{2}$. Some of the severe forms of cutaneous leishmaniasis occur due to host factors, such as elevated Type 2 cytokines and imbalances between tissue inhibitors of metalloproteinase and matrix metalloproteinase ${ }^{3,4}$. Gimblet et al., (2017), proposed that the imbalance of the lesional skin microbiome in cutaneous leishmaniasis wounds caused by L. major plays a major role in altering disease severity and duration ${ }^{5}$. To date, however, relatively few studies have been published on cutaneous leishmaniasis wound microbiome and little information is available on the microbiome of lesions caused by L. donovani.

Patients with chronic ulcerated skin lesions are prone to develop bacterial biofilms which can function as barriers to antibiotic treatment ${ }^{6}$, reduce fibroblast deposition and increase inflammation resulting in impaired wound healing ${ }^{7}$. Biofilms are also known to interact with protozoans in nature ${ }^{8}$. Even though cutaneous leishmaniasis wounds are largely symptomless, a "burning sensation" is common, purportedly reflecting an inflammatory reaction to biofilms ${ }^{9}$. In managing chronic wounds, improved therapeutic modalities could potentially be developed by targeting biofilms ${ }^{10}$ although the current understanding of the involvement of biofilms in cutaneous leishmaniasis wounds is incomplete.

\footnotetext{
${ }^{1}$ Department of Parasitology, Faculty of Medical Sciences, University of Sri Jayewardenepura, Gangodawila, Nugegoda, Sri Lanka. 2Division of Pharmacy and Optometry, School of Health Sciences, Faculty of Biology, Medicine and Health, The University of Manchester, Manchester, UK. ${ }^{3}$ Department of Public Health, Faculty of Medicine, University of Kelaniya, Kelaniya, Sri Lanka. ${ }^{4}$ Department of Anatomy, Faculty of Medical Sciences, University of Sri Jayewardenepura, Gangodawila, Nugegoda, Sri Lanka. ${ }^{5}$ Dermatology Unit, Base Hospital, Tangalle, Sri Lanka. ${ }^{6}$ Department of Family Medicine, Faculty of Medical Sciences, University of Sri Jayewardenepura, Gangodawila, Nugegoda, Sri Lanka. ${ }^{7}$ Department of Pathology, Faculty of Medical Sciences, University of Sri Jayewardenepura, Gangodawila, Nugegoda, Sri Lanka. ${ }^{8}$ Department of Basic Sciences, Faculty of Allied Health Sciences, University of Sri Jayewardenepura, Gangodawila, Nugegoda, Sri Lanka. ${ }^{9}$ Department of Microbiology, Faculty of Medical Sciences, University of Sri Jayewardenepura, Gangodawila, Nugegoda, Sri Lanka. ${ }^{\varpi}$ email: dilhara@sjp.ac.lk
} 
We hypothesized that cutaneous leishmaniasis wounds caused by L. donovani would be associated with distinct microbiome profiles compared to unaffected skin, and that microbial biofilms would form in a proportion of cutaneous leishmaniasis wounds with possible etiological implications. We additionally hypothesized that the microbiome in cutaneous leishmaniasis wounds would differ with lesion size, parasite load and type of lesion.

Thus, we characterized the microbiome of cutaneous leishmaniasis wounds caused by L. donovani comparing it with adjacent and contralateral skin. Also, we visualized and profiled bacterial biofilms in these wounds. The information presented in this manuscript would be useful to gain a better understanding of the cutaneous leishmaniasis microbiome to facilitate future research on wound management.

\section{Results}

Thirty-nine patients were confirmed as cutaneous leishmaniasis positive by PCR. The group included 21 males and 18 females. The mean age was 46.2 years. The investigation included ulcers (wet ulcers $n=10$, dry ulcers $\mathrm{n}=10)$, ulcerated nodules $(\mathrm{n}=13)$, ulcerated papules $(\mathrm{n}=4)$ and ulcerated plaques $(\mathrm{n}=2)$. The mean duration of those wounds at the time of presentation was 16.4 weeks.

The microbiome of cutaneous leishmaniasis wounds caused by Leishmania donovani. DNA was sequenced from 157 samples. Four sites were sampled from 39 patients, including wound biopsies, wound swabs, contralateral skin swabs and adjacent skin swabs. A negative control of purified water was included, which had only 5 reads following quality filtering (raw reads $n=77$ ) and was negligible compared to the high read numbers of the actual samples. This control was, therefore, not subtracted from the analysis.

There were 13,175,234 raw reads of which 3,686,663 were from contralateral skin swabs, 3,462,115 were from adjacent skin swabs, 3,906,256 were from wound swabs and 2,120,200 were from wound biopsies. There were $7,342,254$ quality-filtered sequences with an average of 46,765.95 reads per sample. Each read was approximately $220 \mathrm{bp}$ in size. The reads were allocated to 17,032 operational taxonomic units (OTUs). The mean frequency per OTU was 431.08 .

The taxonomic composition of the data included 51 phyla, 151 classes, 302 orders, 551 families and 1168 genera.

Diversity analysis of the microbiome data of cutaneous leishmaniasis wounds caused by Leishmania donovani. During diversity analysis, data were rarefied to an even depth of 20,000. Thirteen wound biopsy samples and one adjacent skin swab sample, which had a low amount of reads were below this threshold and, hence, not included in diversity analyses.

Alpha-diversity parameters were evaluated for sample richness (Observed OTUs), diversity (Shannon-Weaver diversity) and community evenness (Pielou's index) (Fig. 1a). Statistical significance between each sample type was assessed using the pairwise Kruskal-Wallis test. We found no statistically significant differences in sample richness, Shannon diversity or community evenness between contralateral skin swab and adjacent skin swab samples $(p>0.99)$. Wound swabs and wound biopsies were significantly different to the "healthy" skin swabs (contralateral and adjacent skin swabs, $p<0.001$ ). There was no significant difference found between wound swab and biopsy in terms of diversity and evenness. However, wound swabs had a significantly higher richness (Observed OTUs) compared to wound biopsies.

Unweighted unifraction distance matrix (Fig. 1b) showed clear separation of wound biopsy samples. Wound swabs and biopsies had a significant dissimilarity of bacterial composition compared to contralateral skin swabs and adjacent skin swabs (pairwise Permanova test $p$ value $<0.001$ for all combinations). The healthy skin swabs had similar bacterial compositions (pairwise Permanova test $\mathrm{p}$ value $=0.926$ ).

Relative abundance of the OTUs associated with the cutaneous leishmaniasis microbiome. The top 5 phyla according to the relative abundance in contralateral skin swabs were Actinobacteria (41.55\%), Proteobacteria (23.71\%), Firmicutes (12.92\%), Planctomycetes (8.04\%) and Bacteroidetes (4.23\%). The same 5 phyla were dominant in the adjacent skin swabs. In the wound swabs, Firmicutes were dominant (40.07\%) followed by Actinobacteria (30.48\%), Proteobacteria (18.70\%), Planctomycetes (4.08\%) and Acidobacteria (2.16\%). In wound biopsies, the top 5 phyla were Firmicutes (29.18\%), Acidobacteria (21.81\%), Proteobacteria (20.51\%), Actinobacteria (17.00\%) and Deinococcus-Thermus (4.42\%). Phylum Fusobacteria was generally low in all sample types and relatively absent in wound biopsies. In contralateral skin, adjacent skin and wound swabs, phylum Euryarchaeota and Parvarchaeota (Under Kingdom Archaea), were detected in very low relative abundances $(<0.1 \%)$ and they were frequently absent in wound biopsy samples. Figure 2 shows the relative abundance of the dominant phyla in which the low abundant phyla $(<1 \%$ of relative abundance) are grouped as "Remainder".

DESeq2 analysis showed a significant difference in the relative abundance of 18 OTUs including OTUs belonging to the genera Actinobacter, Planctomyces, Nocardioides, Staphylococcus, Balneimonas and Streptococcus between contralateral skin swabs and wound swabs. An OTU belonging to class Bacilli had a higher relative abundance (statistically significant p.adj $<0.05,20.51 \%)$ in wound swabs than contralateral skin swabs $(0.1 \%)$. Several OTUs $(n=5)$, including Cloacibacterium, were also found to be significantly different in wound swabs compared to adjacent skin swabs (Supplementary Table 1). An OTU belonging to family Ellin6075 was significantly higher in relative abundance in wound swabs (1.24\%) than adjacent skin swabs $(0.07 \%)$. There were 50 OTUs significantly different in relative abundance in wound biopsies compared to wound swabs. In wound biopsies, OTUs of family Ellin6075, Streptococcus, Enhydrobacter, Rubrobacter, Meiothermus were significantly higher in relative abundance compared to wound swabs. Pseudomonas was in higher relative abundance in both 

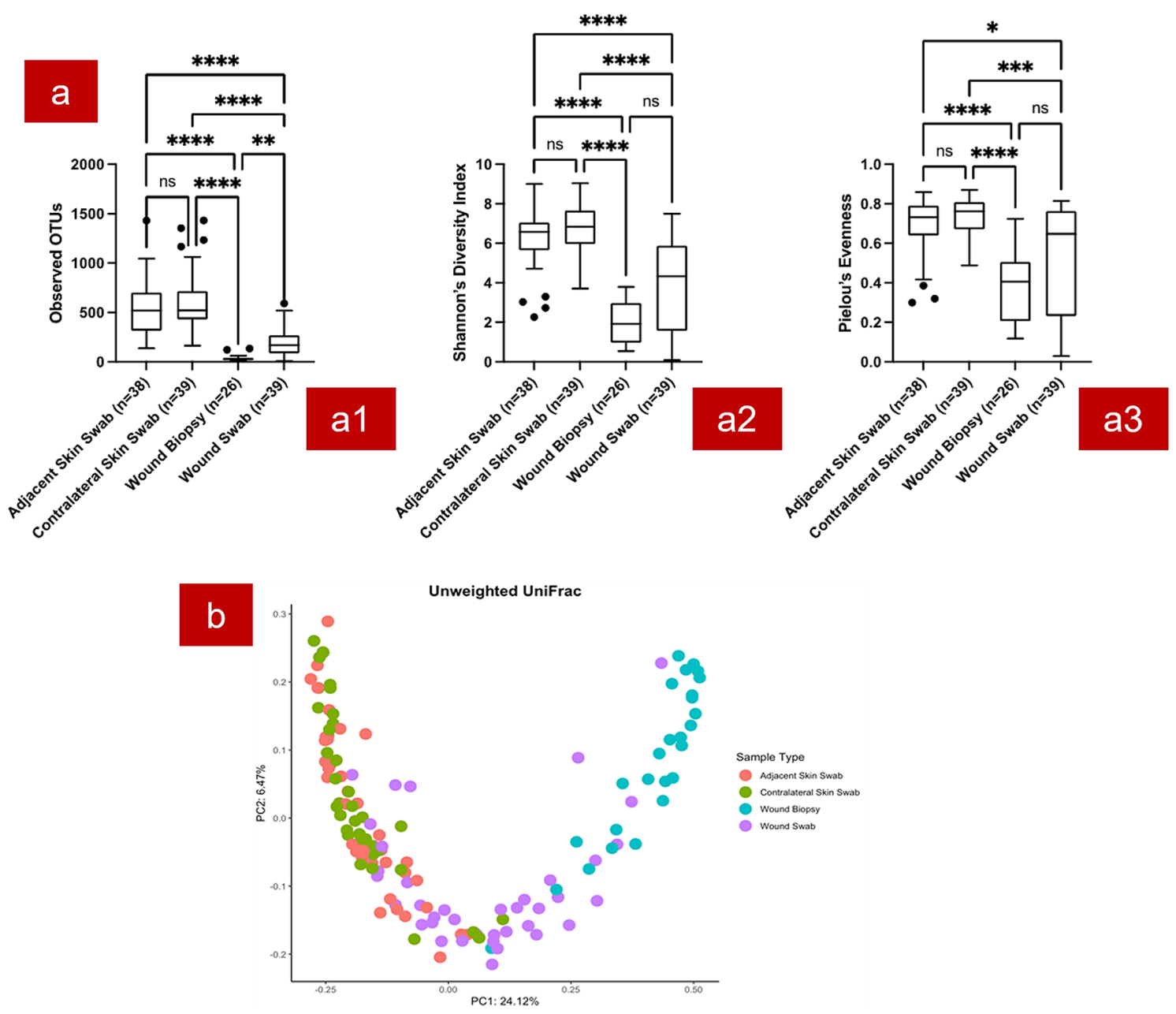

Figure 1. Diversity analysis results of different sample types. (a) Bacterial diversity based on sample location. Observed OTUs (a1); Shannon-Wiener diversity index (a2); Pielou's index (a3); (b) Unweighted Unifraction distance matrix-based ordination ( $\beta$ diversity). Statistical significance of the difference between each sample type is denoted with asterisks. ${ }^{\star} p \leq 0.05 ;{ }^{* *} p \leq 0.01 ;{ }^{* *} p \leq 0.001 ;{ }^{* * *} p \leq 0.0001 ;$ ns: $p>0.05$.

wound swabs (3.34\%) and wound biopsies (4.90\%) than healthy skin (Fig. 3, Supplementary Table 1). Genus Mycobacterium was present in a relative abundance of $0.06 \%$.

Microbiome profile comparison of; parasite load, lesion size and type of cutaneous leishmaniasis lesions. DESeq2 analysis showed that the relative abundance of an OTU of Trabulsiella was significantly higher in lesions with high parasite loads compared to lesions with low parasite loads (p.adj < 0.05$)$ and OTUs belonging to family Ellin6075 and Arcanobacterium spp. were significantly higher in small $(<2 \mathrm{~cm})$ cutaneous leishmaniasis lesions (p.adj <0.05) whereas OTUs of Corynebacterium and class Bacilli were significantly higher in large $(>2 \mathrm{~cm}$ ) lesions (p.adj $<0.05)$.

Alpha and beta diversity assessments of the wound biopsy samples from different types of cutaneous leishmaniasis lesions indicated that there was no significant dissimilarity in terms of microbial composition $(p>0.05$ for the overall Kruskal-Wallis test and the following pairwise Permanova tests for all combinations). DESeq 2 analysis of different lesion types revealed no OTUs significantly different between dry and wet cutaneous leishmaniasis wounds. However, there were 3 OTUs belonging to the genus Enhydrobacter, Bradyrhizobium and Actinomyces which were significantly higher in relative abundance in ulcerative nodules compared to wet ulcers (p.adj $<0.05$ ). Also, an OTU belonging to Corynebacterium was significantly higher in relative abundance in ulcerative nodules compared to dry ulcers (p.adj <0.05) and an OTU belonging to Enhydrobacter was significantly higher in relative abundance in dry ulcers than ulcerative nodules (p.adj < 0.05). Two OTUs belonging to genus Corynebacterium and Micrococcus were significantly higher in relative abundance in wet ulcers compared to ulcerated papules (p.adj <0.05), in which these two OTUs were relatively absent.

Visualizing bacterial biofilms in cutaneous leishmaniasis wounds caused by Leishmania donovani. A pig-skin model was used as a positive control for fluorescence in situ hybridization assay on formalinfixed paraffin-embedded tissue (FFPE-FISH) and Gram staining of formalin-fixed paraffin-embedded tissue 


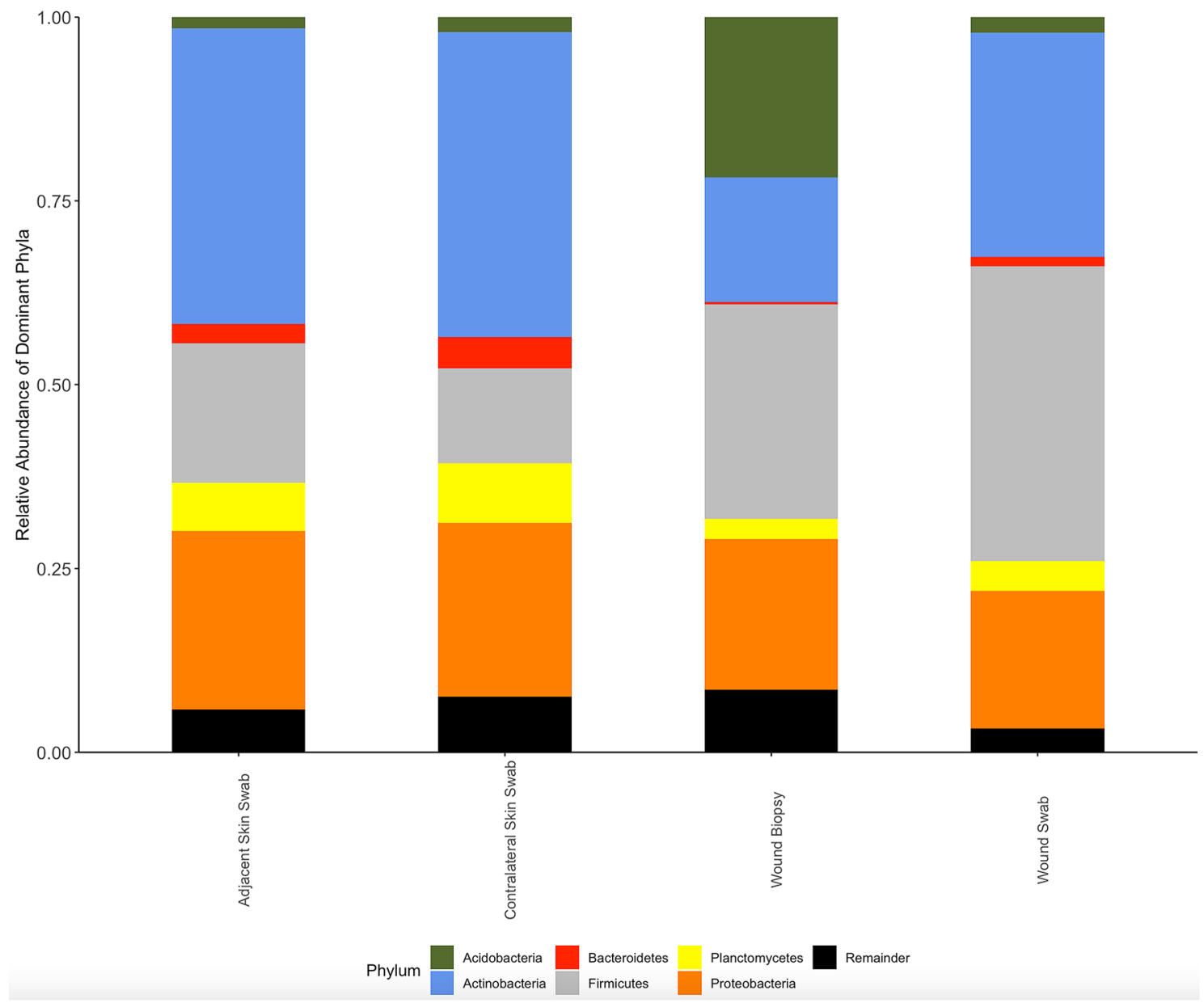

Figure 2. Relative abundance of the phyla in adjacent skin swabs, contralateral skin swabs, wound biopsies, and wound swabs. (Figure generated by R Studio version 1.3.1093 available at http://www.rstudio.com/. Using R version 4.0.3 available at https://www.R-project.org/).

(FFPE-gram) and scanning electron microscopy (SEM) imaging. For the FFPE-FISH section, a CY3 tagged EUB probe was used to stain bacteria red, Concavalin A conjugated Alexa Fluor 488 marked the extracellular polymeric substances in green and DAPI marked the nuclei in blue. For the FFPE-Gram section, bacteria were Gram-stained, and the extracellular polymeric substances was stained orange by Safranin. SEM imaging allowed visualization of both cocci and bacilli (Supplementary Fig. 1).

Wound biopsies of the 39 cutaneous leishmaniases confirmed (by PCR) lesions of patients were visualized for bacterial biofilms. Of them, 24 (61.5\%) lesions were positive for biofilms by FFPE-FISH. By SEM, 23 out of the 39 lesions (59.0\%) were biofilm positive. One sample which was biofilm positive by FFPE-FISH was found to be biofilm negative using SEM. FFPE-Gram detected 14 (35.9\%) lesions as biofilm positive. Fourteen samples were confirmed as biofilm positive and 15 were biofilm negative by all three imaging techniques and 10 were positive at least by one method. The mean size of the biofilms in these cutaneous leishmaniasis lesions was $47.9 \mu \mathrm{m}(\mathrm{SD}+/-32.9$, range $=6.8-141.7)$. There were 2 biofilm lesions $5-10 \mu \mathrm{m}, 13$ lesions $11-50 \mu \mathrm{m}$, and 9 lesions $>50 \mu \mathrm{m}$ in size.

FFPE-FISH, FFPE-Gram and SEM images of cutaneous leishmaniasis wounds are shown in Supplementary Fig. 2, 3a and 3b-c respectively. In some samples, identification of the bacterial red signal by FFPE-FISH was hindered by auto-fluorescent granules of the skin and the extracellular polymeric substances was less (or not) evident. In the FFPE-Gram sections, most of the biofilm positive samples had gram-positive cocci bacterial aggregates. Isolated small aggregates of bacteria were apparent in most of the slides. However, in most of the sections, demonstration of the extracellular polymeric substances and conclusion on biofilm positivity due to the small size of the aggregates was difficult. SEM images of the samples also showed coccoid cells, either in clumps, chains, or both, and the extracellular substance could be demonstrable. Different textures of the extracellular substance could be seen by SEM i.e., smooth, and thin thread-like.

The association of bacterial biofilms in cutaneous leishmaniasis wounds with clinico-demographic characteristics of the study cohort. The association between the presence of biofilm in CL lesions and relevant clinico-demographic variables are summarized in Supplementary Table 2. 


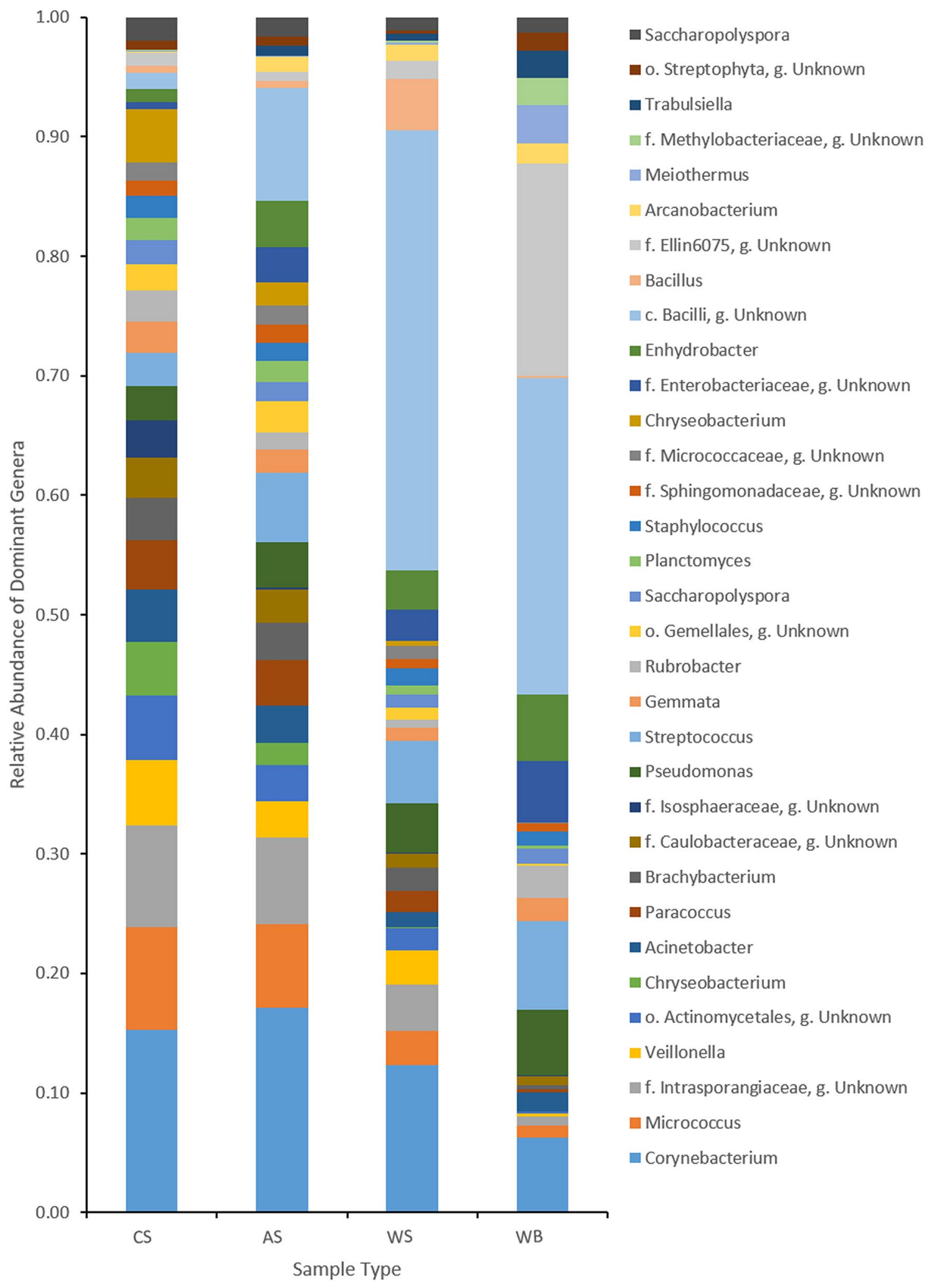

Figure 3. Stacked bar plots with dominant genera (relative abundance $>0.5 \%$ ) in contralateral skin swabs (CS), adjacent skin swabs (AS), wound swabs (WS) and wound biopsies (WB). f: Family; o: Order; g: Genus. (Figure generated by Microsoft Excel 2013). 


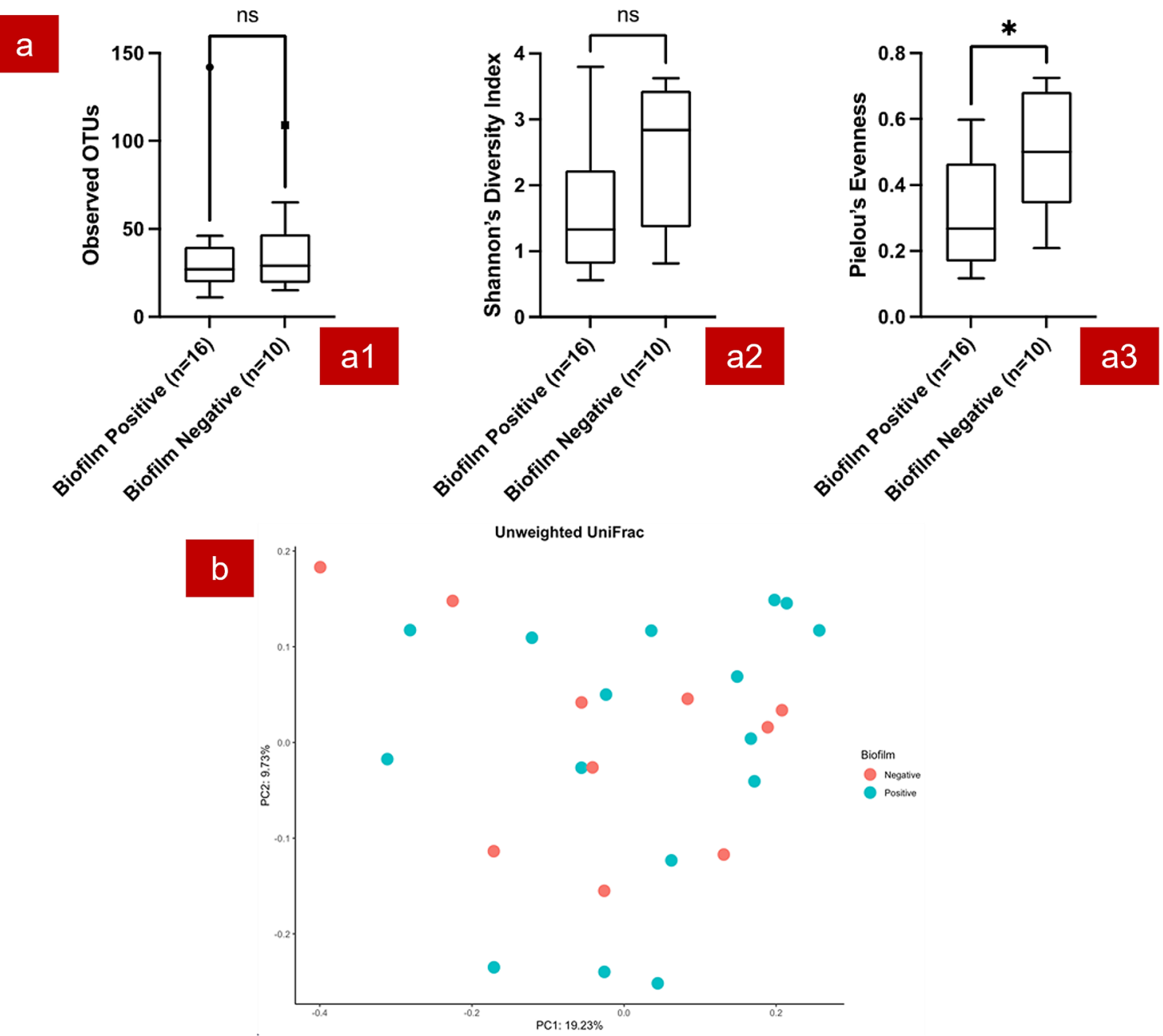

Figure 4. Diversity analysis results of the biofilm positive and negative wound biopsies. (a) Bacterial diversity based on biofilm positivity in wound biopsies. Observed OTUs (a1); Shannon-Wiener diversity index (a2); Pielou's index (a3); (b) Unweighted Unifraction distance matrix based on biofilm positivity of the wound biopsies. ${ }^{*}$ : statistically significant difference $\mathrm{p} \leq 0.05$; ns: no statistically significant difference $p>0.05$.

The mean age of the group was 46.2 years $(S D \pm 16.2$, range $=18-77)$. There was a significant association between biofilm formation and age $(p=0.031)$ with patients over 40 years having a higher percentage of biofilm formation. Wet lesions had a significantly higher biofilm formation as compared to dry lesions $(p=0.004)$. Symptomatic lesions had significantly higher biofilm positivity as compared to asymptomatic lesions $(p=0.015)$. All lesions with pus cell count $\geq 3+$ had biofilms and all the biofilm negatives had a pus cell count of $<3+(p=0.007)$. Biofilm positivity was higher in males (58.3\%), in upper limb lesions $(62.5 \%)$ in CL lesions of $<3$ months $(70.8 \%)$ and in lesions with high parasite counts $(62.5 \%)$ though not significantly different from the respective comparison group.

Profiling of the wound biopsies containing bacterial biofilms. Eight biofilm positive wound biopsy samples and five biofilm negative wound biopsy samples, which had a low amount of reads, did not meet the rarefaction threshold and were excluded from the diversity analysis.

There was no significant difference between the microbiome profiles of "biofilm positive" and "biofilm negative" cutaneous leishmaniasis wound biopsies in terms of alpha diversity except evenness (pairwise Kruskal-Wallis test $\mathrm{p}$ value for Pielou's evenness was 0.023 , Fig. $4 \mathrm{a}$ ). The two groups had no significant difference in terms of beta diversity measurements as well (Pairwise Permanova test $p$ value $>0.05$, Fig. $4 \mathrm{~b}$ ).

DESeq2 analysis showed a significant higher relative abundance of an OTU belonging to class Bacilli (which includes Staphylococcus spp. and Streptococcus spp.) in "biofilm positive" wound biopsies (p.adj<0.05). In addition, almost all the genera which were of $>1 \%$ in "biofilm positive" wound biopsies were biofilm-forming bacteria, and they were in relatively higher abundance in "biofilm positive" wound biopsies compared to "biofilm negative" wound biopsies (Fig. 5).

Further, Figs. 6, 7 and 8 show positive bacterial biofilms (positive by all three imaging techniques), of samples 2, 7, and 27 respectively. In sample 2, class Bacilli was seen at a relative abundance of $89.03 \%$. Whilst the 


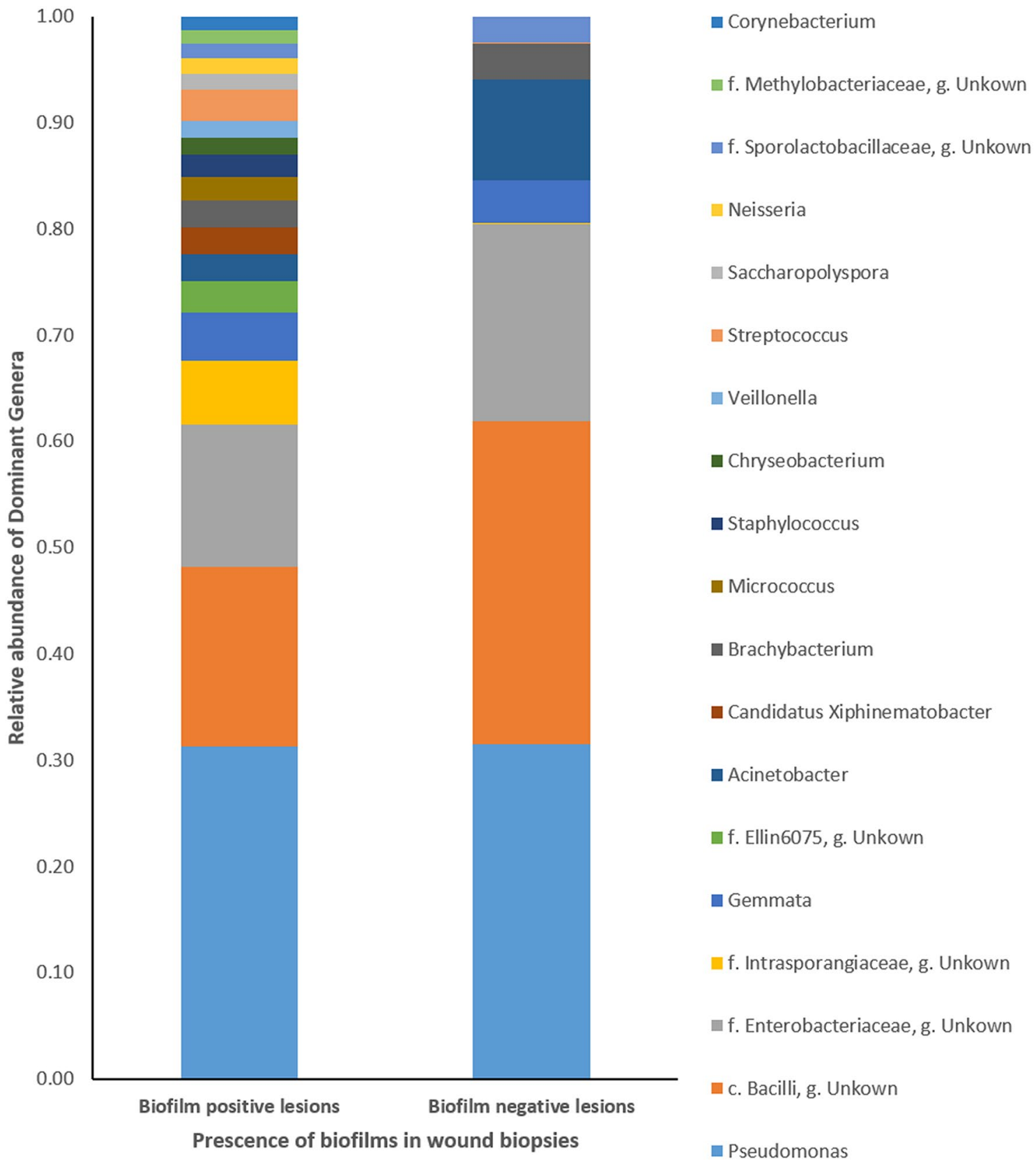

Figure 5. Relative abundance of genera (genera with $>1 \%$ abundance considered) in biofilm positive and negative wound biopsy samples. f: Family; o: Order; g: Genus. (Figure generated by Microsoft Excel 2013).

taxonomic classification of this OTU did not resolve to the level of genus, our initial experiments run with cultures (data not shown), might allow us to infer these cocci in clumps could be Staphylococcus spp. belonging to order Bacillales. Sample 7, for example, found Streptococcus sp. in a relative abundance of $92.34 \%$. In the SEM images the Streptococcus spp. in chains were demonstrable. Sample 27 had Pseudomonas sp. in a relative abundance of $94.07 \%$.

\section{Discussion}

We have profiled the microbiomes of cutaneous leishmaniasis lesions in the presence and absence biofilms. Cutaneous leishmaniasis lesions are chronic in nature. It has been reported that the prevalence of biofilms in non-cutaneous leishmaniasis chronic wounds is more than $78.2 \%{ }^{11,12}$. We detected biofilms in $61.5 \%(24 / 39)$ of the cutaneous leishmaniasis lesions using fluorescence in situ hybridization in formalin-fixed paraffin-embedded tissue (FFPE-FISH). These cutaneous leishmaniasis lesions with biofilms were mainly associated with an OTU belonging to class Bacilli, a class that includes common pyogenic bacteria such as Streptococcus and Staphylococcus, and pseudomonas.

As in a previous study on diabetic wounds ${ }^{13}$, FFPE-FISH detected the most biofilms in cutaneous leishmaniasis lesions followed by SEM. It has been shown that Gram stain often fails to detect biofilms in lesions of 

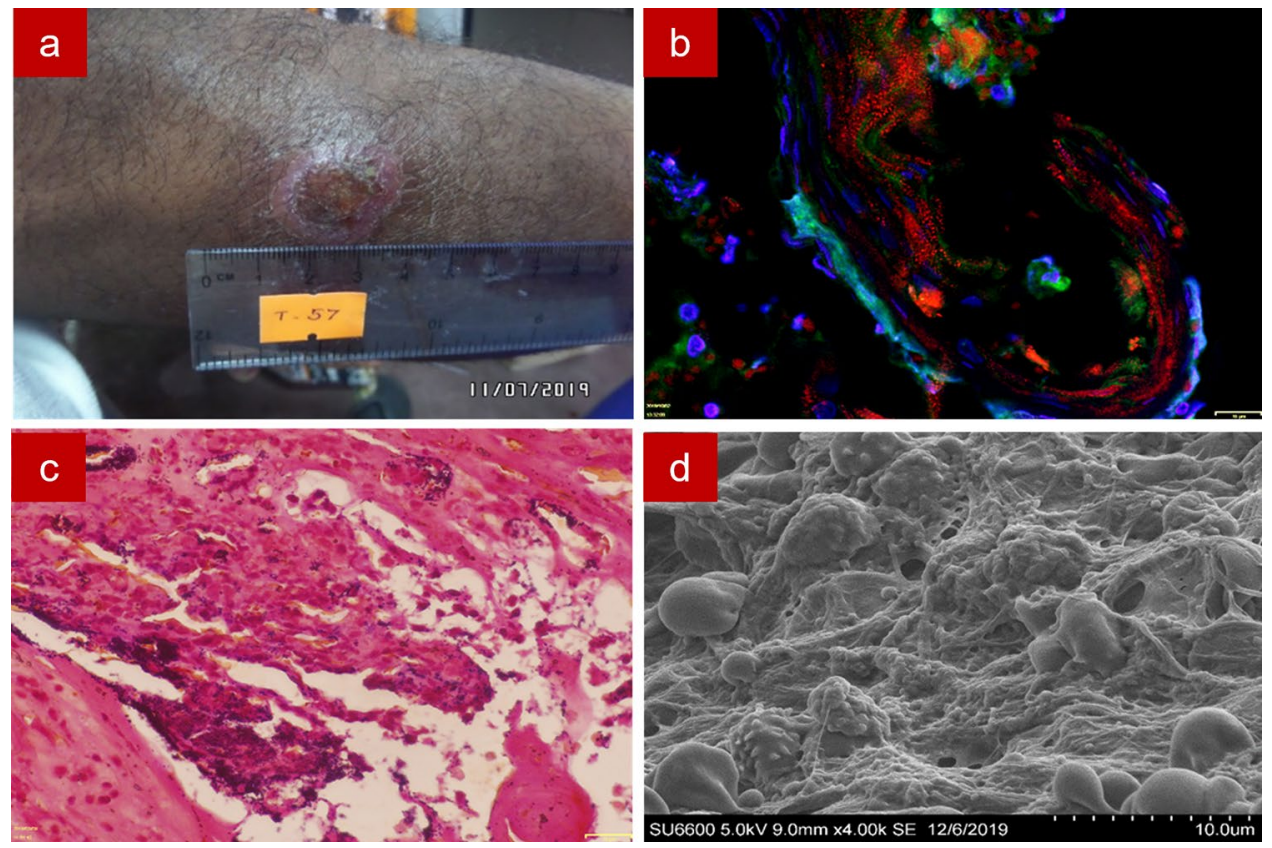

Figure 6. Sample 2. Image of a wet ulcer of 4 weeks duration with a yellow scab in the lower limb (a); Fluorescence in situ hybridization showing bacteria in red with the CY3 tagged Eu bacterial probe, extracellular polymeric substances in green with Concavalin A conjugated Alexa Fluor 488 and tissue nuclei in blue with DAPI (b); Gram staining showing gram-positive cocci in clumps (c); Scanning electron microscopy showing coccoid shaped bacteria embedded in thread-like extracellular polymeric substance (d).
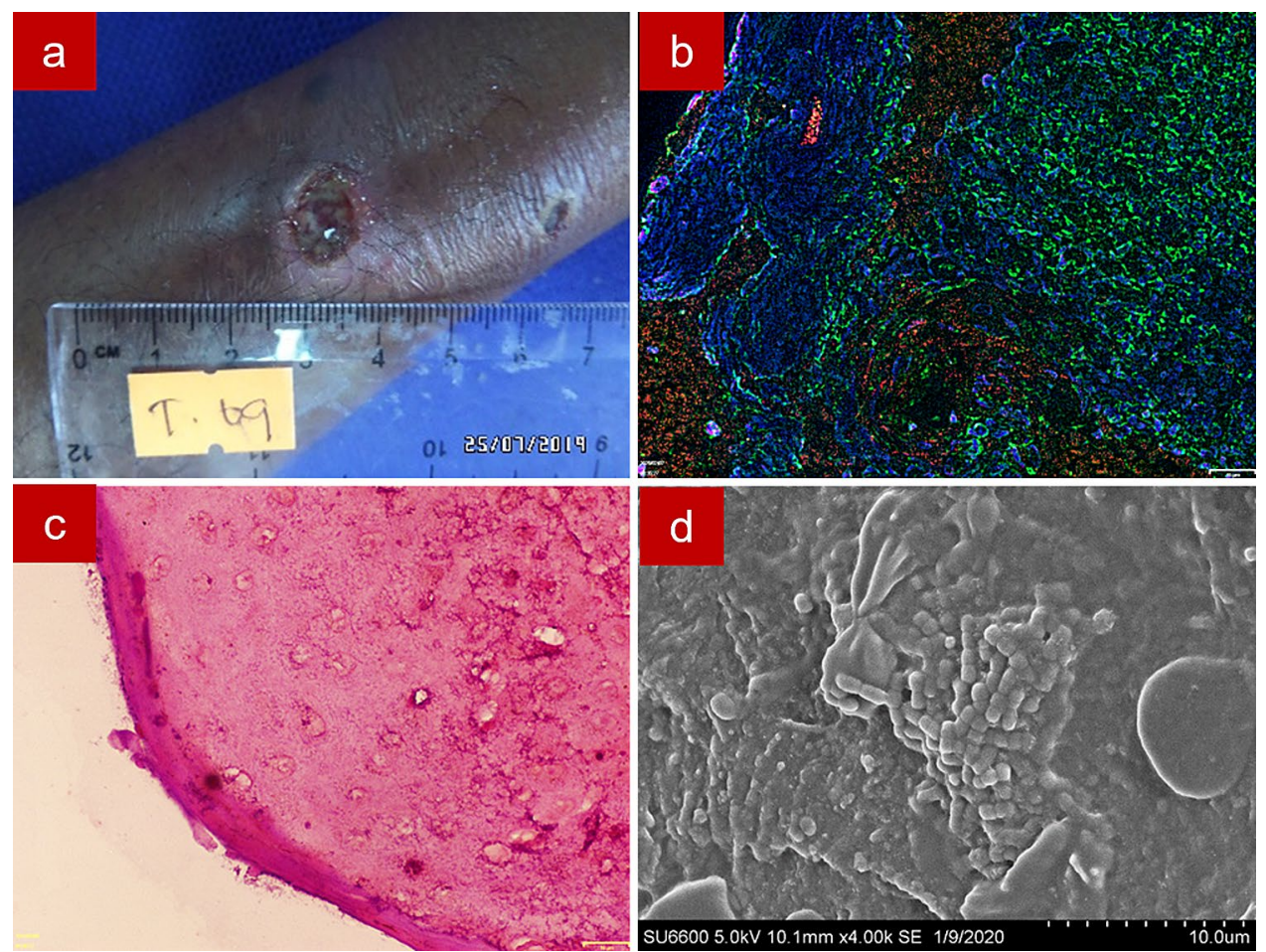

Figure 7. Sample 7. Image of a wet ulcer of 12 weeks duration in the lower limb (a); Fluorescence in situ hybridization showing bacteria in red with the CY3 tagged Eu bacterial probe, extracellular polymeric substances in green with Concavalin A conjugated Alexa Fluor 488 and tissue nuclei in blue with DAPI (b); Gram staining showing gram positive cocci (c); Scanning electron microscopy showing coccoid shaped bacteria in chains embedded in smooth extracellular polymeric substance (d). 

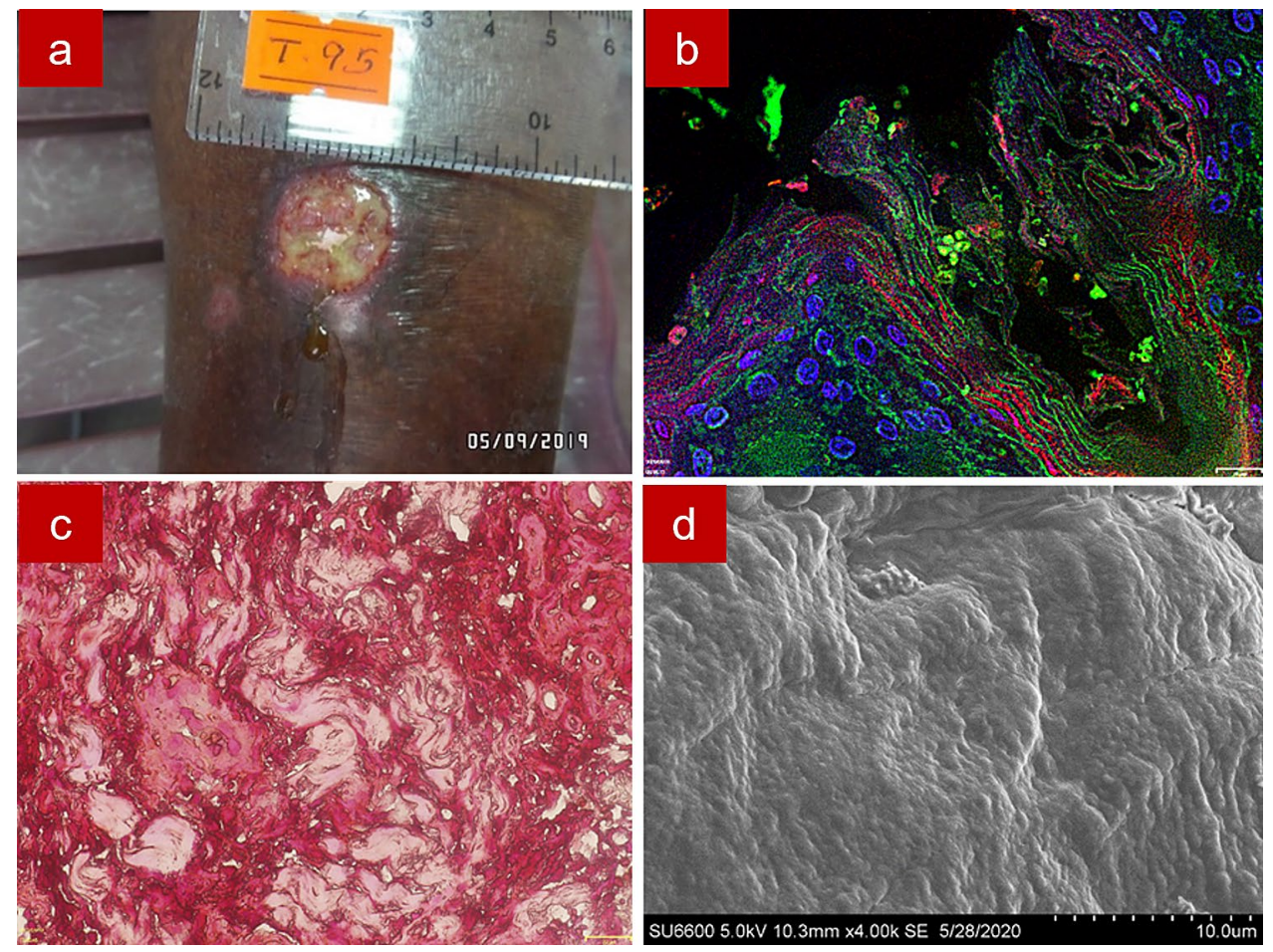

Figure 8. Sample 27. Image of a wet ulcer of 8 weeks duration in the lower limb with green colour pus discharge (a); Fluorescence in situ hybridization showing bacteria in red with the CY3 tagged Eu bacterial probe, extracellular polymeric substances in green with Concavalin A conjugated Alexa Fluor 488 and tissue nuclei in blue with DAPI (b); Gram staining showing gram-negative rods (c); Scanning electron microscopy showing bacteria in embedded in smooth extracellular polymeric substance (d).

less than 1-month duration ${ }^{14}$. In the current study, most of the cutaneous leishmaniasis lesions (59\%) were of less than three months duration and FFPE-Gram failed to detect over $40 \%$ of CL biofilms that were detected by FFPE-FISH. These were wounds with biofilms of small aggregates $(15 / 24$ were $<50 \mu \mathrm{m}$ as measured by FFPEFISH) which may have limited detection.

All lesions with a high pus cell count of $\geq 3+$ with potential infection/ inflammation had biofilms $(p=0.007)$. Gimblet et al., $2017^{5}$ and Salgado et al., $2016^{15}$ have previously suggested that changes in the cutaneous leishmaniasis microbiome might lead to increased inflammation, which could have a downstream effect on disease chronicity and severity in humans.

It has been reported that $60 \%$ of cutaneous leishmaniasis lesions caused by Leishmania braziliensis presented with secretions, $44 \%$ with pain and itching and $40 \%$ with burning sensation ${ }^{9}$. The authors concluded that secretions and burning/itching sensation of cutaneous leishmaniasis lesions are frequently associated with secondary bacterial infection and that the burning sensation may be due to biofilm formation in these lesions. Whilst we did not evaluate burning sensation in the L. donovani-driven cutaneous leishmaniasis wounds, we observed similar percentages of wet (59\%), and symptomatic (pain and/or itching) lesions (33.3\%). Since most of the biofilms in cutaneous leishmaniasis lesions were significantly seen in patients with symptomatic (pain and/or itching) $(p=0.015)$ and wet lesions $(p=0.004)$, the presence of secretions, pain and/or itching may be considered as indicators of biofilm formation.

The bacterial-parasite association in cutaneous leishmaniasis lesions is not well understood. One study showed that co-infection of $S$. aureus and L. major promoted $S$. aureus growth but parasite numbers remained unchanged in murine models ${ }^{16}$. Another in vitro study reported lysis of L. chagasi by S. marcescens SM365 ${ }^{17}$. We did not observe any significant association between biofilm formation and $L$. donovani parasite loads. However, most of the lesions with high parasite load (62.5\%) had biofilms, indicating some change in the microenvironment of these lesions facilitating biofilm formation.

In addition to the description of the biofilms in L. donovani-associated cutaneous leishmaniasis wounds, we assessed the microbiome of the cutaneous leishmaniasis lesions caused by L. donovani. Our results indicate that biopsies and wound swabs from $L$. donovani-associated sites had significantly different alpha and beta diversity profiles to swabs from healthy skin sites. Beyond this, analysis of the OTUs present in each sample type revealed key characteristics of the L. donovani-associated cutaneous leishmaniasis microbiome, in comparison to previously described cutaneous leishmaniasis wounds.

Salgado et. al., 2016 reported that Firmicutes (54.3\%), Actinobacteria (11.7\%) and Fusobacteria (11.6\%) were the top phyla in wound swabs of cutaneous leishmaniasis lesions caused by Leishmania braziliensis ${ }^{15}$ in a Brazilian cohort. In the present study, we observed a considerably lower relative abundance of Firmicutes (40.07\%) 
and higher percentages of Actinobacteria (30.48\%) and Proteobacteria (18.70\%) in wound swabs. In addition to the dominant phyla seen in wound swabs, the wound biopsies showed a low abundance of Deinococcus-Thermus $(4.42 \%)$ which are uncultured thermo-acidophiles adapted to highly resistant environments ${ }^{18}$. In both wound swabs and wound biopsies, Fusobacteria was relatively absent. In contrast to L. braziliensis ${ }^{15}$, we found that aerobic bacteria like Pseudomonas was more common in L. donovani-cutaneous leishmaniasis wounds compared to healthy skin. Facultative anaerobes were also detected in greater abundance than strict anaerobes in our cutaneous leishmaniasis lesions. It has been reported that the presence of facultative anaerobes in wounds act as a poor prognostic factor in the healing of cutaneous wounds ${ }^{19}$. Also, discrete differences, such as the presence of soil-associated bacteria (i.e., Acidobacteria), could be driven by environmental factors of our study population (a high degree of farming) ${ }^{20}$. Organisms such as Proteus spp., Citrobacter spp., Morganella spp. and Propionibacterium spp., which are common in chronic wound microbiome ${ }^{21}$ were relatively low/absent in these cutaneous leishmaniasis lesions suggesting that $L$. donovani associated cutaneous leishmaniasis lesions may have a distinct microenvironment in comparison with other chronic wounds.

Actinobacteria, Firmicutes, Proteobacteria, Bacteroidetes are described as the four dominant phyla of healthy human skin ${ }^{22}$. Similarly, these were the most common in our contralateral skin samples. We also detected a considerable percentage of Planctomycetes (7.46\%) in contralateral skin swabs which is not commonly associated with healthy skin but has been highlighted as a potentially pathogenic organism ${ }^{23}$.

This study evaluated the bacterial profile of cutaneous leishmaniasis wound biopsies. In concordance with the literature on diabetic foot ulcers, the biopsy had significantly lower diversity values compared to swabs ${ }^{24}$. In the cutaneous leishmaniasis wound biopsies, Staphylococcus, Pseudomonas and Streptococcus which are common wound pathogens were detected ${ }^{25}$. Micrococcus, Bacillus spp., and Corynebacterium were also seen in wound biopsies which are usually present in normal skin flora, but with a potential pathogenic role if seen in deep tissues ${ }^{26}$. However, other common wound pathogens such as Enterococcus spp., E. coli, Proteus mirabilis, Bacteroides fragilis were less dominant in these cutaneous leishmaniasis wounds ${ }^{25}$.

Through diversity analysis, we have demonstrated that cutaneous leishmaniasis lesions have significantly different alpha and beta diversities compared to contralateral and adjacent skin. Distinct profiles have previously been described in cutaneous leishmaniasis lesions caused by L. major ${ }^{5}$ and L. braziliensis $^{15}$, with evidence that lesion microbiomes are transmissible to the adjacent skin in murine models ${ }^{5}$. Here, we did not find any significant similarity in microbiomes of the cutaneous leishmaniasis lesion and adjacent skin of $1 \mathrm{~cm}$ distant to the lesion. Whilst Gimblet et al., 2017 detected this transmission of lesion microbiome constituents to adjacent skin after 12 weeks post-infection ${ }^{5}$, our group of patients mostly (59\%) had lesions of $\leq 3$ months duration. Therefore, if transmission does occur it is possibly undetectable at an earlier sampling stage.

We assessed the microbiome of different types of lesions. In addition, we statistically compared the microbiomes of small and large lesions, and lesions with high and low parasite loads. We found that even very small cutaneous leishmaniasis lesions (including ulcerated papules) had well-developed biofilms. However, ulcerated papules lacked potentially pathogenic OTUs, such as genus Corynebacterium and Micrococcus, compared to wet ulcers. OTUs of Corynebacterium and class Bacilli were significantly higher in relative abundance in wound biopsies of large lesions and were also significantly higher in lesions with biofilms. Even though the size of the lesion was not significantly associated with biofilm formation, these potentially pathogenic biofilms might have influenced the lesion size. An OTU belonging to the genus of Trabulsiella was significantly higher in relative abundance in wound biopsies of lesions with high parasite loads. This genus can be a potentially opportunistic pathogen of faecal origin ${ }^{27}$.

In conclusion, our results indicate that Leishmania donovani-associated cutaneous leishmaniasis wounds have a distinct, less diverse, microbiome than adjacent and contralateral skin. The microbiome of the wound biopsies included common wound pathogens (Staphylococcus, Pseudomonas and Streptococcus) and potentially pathogenic organisms (Micrococcus, Bacillus spp., and Corynebacterium). Greater abundances of potentially pathogenic organisms were observed in wet ulcers, lesions with high parasite loads and large wounds. More than half of the wounds (61.5\%) had bacterial biofilms significantly associated with an OTU belonging to class Bacilli. All lesions with a high pus cell count ( $\geq 25 /$ low power filed), $79.2 \%$ of the wet lesions and $92.3 \%$ of the symptomatic lesions had significantly higher biofilm positivity $(p$ value $<0.05)$.

\section{Methods}

All methods were conducted in accordance with relevant guidelines and regulations.

Study design. A cross-sectional study was conducted at Base Hospital Tangalle, Southern Province, Sri Lanka from July 2019 to October 2020 (cutaneous leishmaniasis is endemic to this location and the only identified species is L. donovani). Patients above 18 years with highly suspicious clinical ulcerated lesions, giving informed written consent were included. Exclusion criteria included: any person with debilitating illness or immunosuppression, or had previously received standard treatment for cutaneous leishmaniasis for the same presenting lesion, or recently (within the last 6 months) visited a cutaneous leishmaniasis endemic country (to exclude the possibility of including cutaneous leishmaniasis lesions caused by other species), had lesions at sites from which punch biopsies could not be taken (i.e., eyelid, pinna of the ear), received antibiotic treatment or herbal applications during the past 2 weeks ${ }^{28}$, or used "Lifebuoy" soap product (proven to be bactericidal and is used commonly in Sri Lanka ${ }^{29}$ ), did not have ulcerated lesions ${ }^{30}$, were laboratory-confirmed to have Diabetes mellitus with $\mathrm{HbAlc}$ level $>6.5 \%{ }^{31}$, or patients with other skin disorders which are a major confounding factor in wound and microbiome investigations.

An interviewer-administered case report form was used to gather demographic and clinical data. 
Sample size calculation. A minimum sample size of 36 was required to estimate the proportion of cutaneous leishmaniasis lesions having a biofilm as $30 \%$ (based on preliminary work) with an alpha error of 0.05 and an acceptable difference of 0.15 . A consecutive sample of 39 individuals with cutaneous leishmaniasis lesions satisfying inclusion and exclusion criteria was investigated for the presence of biofilms. The same number of patients were included in microbiome analysis as well.

Sample collection. In addition to the below-mentioned samples, two slit skin smears, an impression smear prepared from the wound biopsy and a $2 \mathrm{ml}$ venous blood sample (for high proficiency liquid chromatographic HbA1c assay to exclude Diabetes mellitus) were collected.

Skin swabs and wound swabs. Three swabs were collected from contralateral skin, adjacent skin and wound before cleaning ${ }^{22}$. The wound scab was removed before the collection of the wound swab. An adjacent skin swab was taken $1 \mathrm{~cm}$ away from the lesion edge ${ }^{32}$. Sterile COPAN Flocked swabs were pre-moistened in a solution containing $50 \mathrm{mM}$ Tris, $1 \mathrm{mM}$ EDTA, $0.5 \%$ Tween 20 . Contralateral and adjacent skin swabs were taken following rubbing an area of $4 \mathrm{~cm}^{2}, 50$ times $(30 \mathrm{~s})$ with pressure ${ }^{33}$. The swab head was cut with sterile scissors and put directly into MO BIO Power soil bead tube (QIAGEN, Germany). This was transported in ice and stored in $-20{ }^{\circ} \mathrm{C}$ till DNA extraction (maximum storage time was $24 \mathrm{~h}$ ).

Collection of wound biopsies. A full-thickness wound biopsy $(3-5 \mathrm{~mm})$ from the wound edge with a catchment of the wound base was obtained from each patient under aseptic conditions following cleaning with Povidone Iodine. Aseptic conditions were maintained as obtaining a biopsy can introduce infections to the exciting wound. The wound edge was sampled instead of only the wound base because the Leishmania parasites aggregate at the lesion edge?.

Each biopsy sample was divided into three sections. One section of the wound biopsy (Section A) was transported in MO BIO Power Soil bead tube (QIAGEN, Germany), in ice and immediately transferred to $-20^{\circ} \mathrm{C}$ until DNA extraction (maximum storage time was $24 \mathrm{~h}$ ). The second section (Section B) was transported in $10 \%$ Buffered Neutral Formalin ${ }^{34}$ and fixed for $24 \mathrm{~h}$ at room temperature ${ }^{35}$. The third section (Section C) was transported in $2.5 \%$ Glutaraldehyde with $0.1 \mathrm{M}$ phosphate buffer and stored at $4^{\circ} \mathrm{C}^{36}$.

Laboratory protocols of the study. DNA extracts from section A of the wound biopsy were used for the disease confirmation by a previously described 18S PCR with LITSR/L5.8S primers targeting the ITS1 region of Genus Leishmania with a sensitivity of $92.1 \%$ and specificity of $100 \%$ to detect $L$. donovani ${ }^{37}$.

The 2 slit skin smears were Giemsa stained and examined under oil immersion for Leishmania amastigotes. Parasite load calculation in these smears was done as described in the literature ${ }^{38}$.

DNA extracts of the swabs (4.4.1) and section A of the wound biopsy (4.4.2) were subjected to microbiome analysis by NGS (4.4.3).

Three different imaging techniques were used to visualize biofilms. Section B of the wound biopsy was histopathologically processed to make formalin-fixed paraffin-embedded tissues (FFPE) and subjected to Gram staining (Gram-FFPE, 4.4.4) and fluorescence in situ hybridization (FISH-FFPE, 4.4.5). Section C of the wound biopsy was subjected to scanning electron microscopy (SEM, 4.4.6). There is no gold standard in the detection of biofilms to date ${ }^{39}$. Hence, in this study, we used FISH as the reference standard in confirming biofilms of these cutaneous leishmaniasis lesions ${ }^{39}$ and the rest as supportive evidence. A pig skin model was prepared for biofilm controls ${ }^{40}$. If 2 of 3 criteria (evidence of bacterial attachment to a surface, aggregations of bacteria (microcolony formation) and presence of extracellular polymeric substances) were present, the presence of biofilm was confirmed ${ }^{13}$. Bacterial aggregations measuring $>5 \mu \mathrm{m}$ were considered under the second criterion ${ }^{32}$.

The impression smear was gram stained to calculate the pus cell count. Pus cell count of $\geq 3+(\geq 25$ pus cells/ low power field) was taken to confirm the presence of potential infection/inflammation ${ }^{41}$.

DNA extraction. Extractions were performed using DNeasy Power Soil bead tube (QIAGEN, Germany) according to manufacturer's guidelines ${ }^{42}$ with modifications as suggested in the literature ${ }^{43}$. C1 to C6 are solutions that come with the extraction kit.

The power bead tube with the swab head was vortexed briefly before adding $60 \mu \mathrm{l}$ of $\mathrm{C} 1$. The tubes were then vortexed horizontally for $15 \mathrm{~min}$ and incubated at $70^{\circ} \mathrm{C}$ for $15 \mathrm{~min}$. Following centrifuging, the supernatant was transferred to a collection tube and $250 \mu$ of $\mathrm{C} 2$ was added. This was incubated at $4{ }^{\circ} \mathrm{C}$ for 5 min and centrifuged. The same procedure was repeated following the addition of $200 \mu \mathrm{l}$ of C3. The supernatant was transferred to a new collection tube and $1200 \mu \mathrm{l}$ of C4 was added. Following vortexing, the total volume was transferred as $675 \mu \mathrm{l}$ aliquots to the spin column and centrifuged discarding the flow-through. $500 \mu \mathrm{l}$ of $\mathrm{C} 5$ was added to the drum and centrifuged. DNA was yielded in $50 \mu \mathrm{l}$ of C6.

DNA extraction from section A of the wound biopsy. This was done as described above with the flowing modifications mentioned ${ }^{44}$.

The biopsy tissue was chopped using sterile blades and added to the same bead tube. It was bead beat, horizontally for $1 \mathrm{~min}$ for homogenization of tissue and incubated at $56^{\circ} \mathrm{C}$ for $20 \mathrm{~min}$ following the addition of $\mathrm{C} 1$ and $20 \mu \mathrm{l}$ of Proteinase K (QIAGEN, Germany).

Illumina MiSeq amplicon sequencing. Paired-end amplicon sequencing was carried out at the Centre for Genomic Research, University of Liverpool, Crown Street, Liverpool, L69 7ZB, United Kingdom. Sequencing 
protocol was carried out as described in Illumina $16 \mathrm{~S}$ library preparation guide with Illumina primers with overhang adaptor sequences targeting 16S V3 and V4@@region ${ }^{45}$.

Forward Primer: 5’TCGTCGGCAGCGTCAGATGTGTATAAGAGACAGCCTACGGGNGGCWGCAG. Reverse Primer: 5'GTCTCGTGGGCTCGGAGATGTGTATAAGAGACAGGACTACHVGGGTATCTAATCC.

FFPE-Gram (section B of the wound biopsy) to visualize bacterial biofilms. Routine Gram staining was done following deparaffinization of FFPE tissue sections. Safranin was used to stain the extra polymer matrix of bacterial biofilms ${ }^{46}$. Slides were examined under a bright field of an Olympus FSX100 microscope $(\times 40)$.

FFPE-FISH (section B of the wound biopsy) to visualize bacterial biofilms. A Cyanine 3 tagged Eu-bacterial probe (EUB 338 5'Cy3-GCT GCC TCC CGT AGG AGT3') was used to mark the bacteria (Excitation-550 nm, Emission-570 nm $)^{13}$. The probe was synthesized at Integrated DNA Technologies, USA. Probe dilution was done in hybridization buffer ( $50 \%$ Formamide, $0.9 \mathrm{M} \mathrm{NaCl}, 20 \mathrm{mM}$ TrisHCl (pH 7.6), 0.01\% Sodium Dodecyl Sulfate $(\mathrm{w} / \mathrm{v})$; each $10 \mu \mathrm{l}$ of hybridization buffer contained $50 \mathrm{ng}$ of probe $)^{13}$.

For enzymatic lysis, Lysozyme buffer was prepared (100 mM TrisHCl (pH 8), $50 \mathrm{mM}$ Ethylenediaminetetraacetic acid, and $5 \mathrm{mg} / \mathrm{ml}$ Lysozyme (Sigma Aldrich) ${ }^{13}$.

For staining of the EPS of the biofilms, ConcanavalinA conjugated Alexa Fluor 488 (Excitation-495 nm, Emission-643 nm) (Invitrogen, USA) was used at a concentration of $1 \mathrm{mg} / \mathrm{ml}^{13}$.

The hybridization procedure was carried out as previously described with the following modifications ${ }^{47}$. For enzymatic lysis, Lysozyme buffer was applied $(5 \mu \mathrm{l})$ and incubated in a humid chamber at $45^{\circ} \mathrm{C}$ for $4 \mathrm{hrs}{ }^{13}$. Following post hybridization washing, the air-dried sample was subjected to staining with ConcanavalinA conjugated Alexa Fluor 488 for $1 \mathrm{~h}$ at room temperature.

Several sections of processed tissues were examined under an Olympus FSX 100 microscope $(\times 40)$. All samples were assessed and interpreted independently by two individuals and confirmed by a third investigator. Measurement of the diameter of the largest biofilm in each image $e^{30}$ and image enhancement was done using Image J softwre (Fuji version) $)^{48}$.

Scanning electron microscopy to visualize bacterial biofilms. The fixed biopsy tissue was dehydrated in a series of Ethanol ${ }^{36}$. The tissues were imaged using a Hitachi SU6600 Analytical Variable Pressure Field Emission Scanning Electron Microscope at $5 \mathrm{kV}$ after gold sputter coating of the sample for $15 \mathrm{~s}$.

Statistical analysis. Bioinformatics analyses. Paired-end sequencing resulted in forward and reverse FASTQ files. These were trimmed for the presence of Illumina adapter sequences using Cutadapt version 1.2.1 ${ }^{49}$. The option -O 3 was used, so the 3' end of any reads which match the adapter sequence for $3 \mathrm{bp}$. or more were trimmed. The reads were further trimmed using Sickle version 1.200 with a minimum window quality score of 20. Reads shorter than $15 \mathrm{bp}$ after trimming were removed ${ }^{50}$. Quality filtering and downstream sequence analysis were performed using Qiime 2-2020.2 pipeline according to the Standard Operating Procedure (SOP) available on https://docs.qiime2.org/2020.6/tutorials/moving-pictures $/{ }^{51}$. The OTUs were clustered at $97 \%$ sequence similarity and taxonomy was applied by a Naïve Bayes classifier trained on this dataset using reference sequences from the latest Greengenes database (v13_8) ${ }^{52}$. Alpha diversity was calculated in QIIME2, statistical testing and plotting of figures were performed using GraphPad Prism version 9.2.0 for macOS, GraphPad Software, San Diego, California USA, www.graphpad.com. Beta diversity results was imported from QIIME2 and plotted using QIIME2R ${ }^{53}$, phyloseq ${ }^{54}$, tidyverse ${ }^{55}$ and ggplot $2^{56}$. DESeq2 testing was performed, with the calculation of geometric means prior to estimation of size factors, in the same software, using the DESeq2 package ${ }^{57}$, QIIME2R ${ }^{53}$, phyloseq ${ }^{54}$ and ggplot $2^{56}$.

Data analysis of biofilm investigation. Statistical analysis of the biofilm investigation was done using R Version 4.0.1. Chi-square test with Yates' continuity correction and Fisher's Exact Test were used to test for significant associations. In all cases, biofilm positivity was confirmed by three independent reviewers. Age of the participants was grouped as $<40$ and $>40$ years. CL lesions (ulcers, ulcerated nodules, ulcerated papules and ulcerated plaques) were categorized as wet or dry lesions. Wet lesions had pus/sero-purulent discharge. The maximum diameter of each lesion was categorized as $<2 \mathrm{~cm}$ and $>2 \mathrm{~cm}^{58}$. All the lesions were categorized as $<3$ months or $>3$ months of duration at the time of presentation ${ }^{59}$. CL lesions are usually asymptomatic; lesions with pain and/or itching at the time of presentation were categorized as symptomatic lesions. Parasite load as assessed by Giemsa-stained slit skin smears were categorized into 2 groups; lesions with a parasite load of nil or $1+$ were grouped as "low parasite load" and lesions with a parasite load of $2+$ to 6 + were grouped as "high parasite load"60. The size of the largest biofilm ${ }^{61}$ in each lesion was categorized as $5-10 \mu \mathrm{m}, 11-50 \mu \mathrm{m}$ and $>50 \mu \mathrm{m}^{62}$.

Ethical approval. Ethics approval was obtained from the Ethics Review Committee, Faculty of Medical Sciences, University of Sri Jayewardenepura, Sri Lanka (number 27/19).

\section{Data availability}

http://www.ncbi.nlm.nih.gov/bioproject/781195 (BioProject ID PRJNA781195).

Received: 11 August 2021; Accepted: 15 November 2021

Published online: 30 November 2021 


\section{References}

1. Kaluarachchi, T. D. J. et al. Diagnosing cutaneous leishmaniasis using fluorescence in situ hybridization: The Sri Lankan perspective. Pathog. Glob. Health 113, 180-190. https://doi.org/10.1080/20477724.2019.1650228 (2019).

2. Burza, S., Croft, S. L. \& Boelaert, M. Leishmaniasis. Lancet 392, 951-970. https://doi.org/10.1016/s0140-6736(18)31204-2 (2018).

3. Maretti-Mira, A. C., Rodrigues, K. M. D. P., Oliveira-Neto, M. P. D., Pirmez, C. \& Craft, N. MMP-9 activity is induced by Leishmania braziliensis infection and correlates with mucosal leishmaniasis. Acta Trop. 119, 160-164. https://doi.org/10.1016/j.actatropica. 2011.05.009 (2011).

4. Campos, T. M. et al. Matrix metalloproteinase 9 production by monocytes is enhanced by TNF and participates in the pathology of human cutaneous Leishmaniasis. PLoS Neglect. Trop. Dis. 8, e3282. https://doi.org/10.1371/journal.pntd.0003282 (2014).

5. Gimblet, C. et al. Cutaneous Leishmaniasis induces a transmissible dysbiotic skin microbiota that promotes skin inflammation. Cell Host Microbe 22, 13-24.e4. https://doi.org/10.1016/j.chom.2017.06.006 (2017).

6. Stewart, P. S. Mechanisms of antibiotic resistance in bacterial biofilms. Int. J. Med. Microbiol. 292, 107-113. https://doi.org/10. 1078/1438-4221-00196 (2002).

7. Metcalf, D. \& Bowler, P. Biofilm delays wound healing: A review of the evidence. Burns Trauma 1, 5. https://doi.org/10.4103/ 2321-3868.113329 (2013).

8. Arndt, H., Schmidt-Denter, K., Auer, B. \& Weitere, M. Protozoans and biofilms. In Fossil and Recent Biofilms 161-179 (2003). https://doi.org/10.1007/978-94-017-0193-8_10.

9. Antonio, L. D. F. et al. Effect of secondary infection on epithelialisation and total healing of cutaneous leishmaniasis lesions. Mem. Inst. Oswaldo Cruz 112, 640-646. https://doi.org/10.1590/0074-02760160557 (2017).

10. Percival, S. L., Mccarty, S. M. \& Lipsky, B. Biofilms and wounds: An overview of the evidence. Adv. Wound Care 4, 373-381. https:// doi.org/10.1089/wound.2014.0557 (2015).

11. Malone, M. et al. The prevalence of biofilms in chronic wounds: A systematic review and meta-analysis of published data. J. Wound Care 26, 20-25. https://doi.org/10.12968/jowc.2017.26.1.20 (2017).

12. Dilhari, A. et al. Biofilm prevalence and microbial characterisation in chronic wounds in a Sri Lankan cohort. Lett. Appl. Microbiol. 73, 477-485 (2021).

13. Oates, A. et al. The Visualization of biofilms in chronic diabetic foot wounds using routine diagnostic microscopy methods. J. Diabetes Res. 2014, 1-8. https://doi.org/10.1155/2014/153586 (2014).

14. James, G. A. et al. Biofilms in chronic wounds. Wound Repair Regener. 16, 37-44. https://doi.org/10.1111/j.1524-475x.2007.00321.x (2008).

15. Salgado, V. R. et al. The microbiological signature of human cutaneous leishmaniasis lesions exhibits restricted bacterial diversity compared to healthy skin. Mem. Inst. Oswaldo Cruz 111, 241-251. https://doi.org/10.1590/0074-02760150436 (2016).

16. Borbón, T. Y. et al. Coinfection with Leishmania major and Staphylococcus aureus enhances the pathologic responses to both microbes through a pathway involving IL-17A. PLoS Neglect. Trop. Dis. 13, e0007247. https://doi.org/10.1371/journal.pntd.00072 47 (2019).

17. Moraes, C. S. et al. Leishmania (Leishmania) chagasi interactions with Serratia marcescens: Ultrastructural studies, lysis and carbohydrate effects. Exp. Parasitol. 118, 561-568. https://doi.org/10.1016/j.exppara.2007.11.015 (2008).

18. Theodorakopoulos, N., Bachar, D., Christen, R., Alain, K. \& Chapon, V. Exploration of Deinococcus-Thermus molecular diversity by novel group-specific PCR primers. MicrobiologyOpen https://doi.org/10.1002/mbo3.119 (2013).

19. Verbanic, S., Shen, Y., Lee, J., Deacon, J. M. \& Chen, I. A. Microbial predictors of healing and short-term effect of debridement on the microbiome of chronic wounds. npj Biofilms Microbiomes https://doi.org/10.1038/s41522-020-0130-5 (2020).

20. Prescott, S. \& Logan, A. Transforming life: A broad view of the developmental origins of health and disease concept from an ecological justice perspective. Int. J. Environ. Res. Public Health 13, 1075. https://doi.org/10.3390/ijerph13111075 (2016).

21. Society, M. Microbial communities within the chronic wound. Microbiology Society Available at: https://microbiologysociety.org/ publication/past-issues/the-microbiome/article/microbial-communities-within-the-chronic-wound.html. (accessed 6 July 2021).

22. Grice, E. A. \& Segre, J. A. The skin microbiome. Nat. Rev. Microbiol. 9, 244-253. https://doi.org/10.1038/nrmicro2537 (2011).

23. Aghnatios, R. \& Drancourt, M. Gemmataspecies: Planctomycetes of medical interest. Future Microbiol. 11, 659-667. https://doi. org/10.2217/fmb-2015-0001 (2016).

24. Travis, J. et al. The microbiome of diabetic foot ulcers: a comparison of swab and tissue biopsy wound sampling techniques using 16S rRNA gene sequencing. BMC Microbiol. https://doi.org/10.1186/s12866-020-01843-2 (2020).

25. What are the pathogens commonly associated with wound infections? Latest Medical News, Clinical Trials, Guidelines-Today on Medscape (2021). Available at: https://www.medscape.com/answers/188988-82335/what-are-the-pathogens-commonly-assoc iated-with-wound-infections. (accessed 6 July 2021).

26. Heravi, F. S., Zakrzewski, M., Vickery, K., Armstrong, D. G. \& Hu, H. Bacterial diversity of diabetic foot ulcers: Current status and future prospectives. J. Clin. Med. 8, 1935. https://doi.org/10.3390/jcm8111935 (2019).

27. Mahon, C. R. \& Lehman, D. C. Textbook of Diagnostic Microbiology (Elsevier Saunders, 2019).

28. Kalan, L., Zhou, M., Labbie, M. \& Willing, B. Measuring the microbiome of chronic wounds with use of a topical antimicrobial dressing-A feasibility study. PLoS ONE https://doi.org/10.1371/journal.pone.0187728 (2017).

29. Kaliyadan, F., Aboulmagd, E. \& Amin, T. Antimicrobial activity of commercial "antibacterial" handwashes and soaps. Indian Dermatol. Online J. 5, 344. https://doi.org/10.4103/2229-5178.137799 (2014).

30. Høiby, N. et al. ESCMID* guideline for the diagnosis and treatment of biofilm infections 2014. Clin. Microbiol. Infect. https://doi. org/10.1016/j.cmi.2014.10.024 (2015).

31. (PDF) Diabetes Mellitus: Glucose Control. Available at: https://www.researchgate.net/publication/257187732_Diabetes_Mellitus_ Glucose Control. (accessed 6 July 2021).

32. Bay, L. et al. Bacterial aggregates establish at the edges of acute epidermal wounds. Adv. Wound Care 7, 105-113. https://doi.org/ 10.1089/wound.2017.0770 (2018).

33. Manual of Procedures for Human Microbiome Project, Version 9.0. National Center for Biotechnology Information Available at: https://www.ncbi.nlm.nih.gov/projects/gap/cgi-bin/document.cgi?study_id=phs000228.v3.p1\&phd=2235. (accessed 6 July 2021).

34. Formalin Fixative - Surrey. Available at: https://www.surrey.ac.uk/sites/default/files/Formalin-Fixative.pdf. (accessed 6 July 2021 ).

35. Petersen, B. L., Sørensen, M. C., Pedersen, S. \& Rasmussen, M. Fluorescence in situ hybridization on formalin-fixed and paraffinembedded tissue. Appl. Immunohistochem. Mol. Morphol. 12, 259-265. https://doi.org/10.1097/00129039-200409000-00013 (2004).

36. Failure Analysis and Scanning Electron Microscopy. SEM Lab Inc. (2021). Available at: https://www.semlab.com/. (accessed 6 July 2021).

37. Ranasinghe, S. et al. Polymerase chain reaction detection of LeishmaniaDNA in skin biopsy samples in Sri Lanka where the causative agent of cutaneous leishmaniasis is Leishmania donovani. Mem. Inst. Oswaldo Cruz 110, 1017-1023. https://doi.org/10.1590/ 0074-02760150286 (2015).

38. Chulay, J. D. \& Bryceson, A. D. M. Quantitation of amastigotes of Leishmania Donovani in smears of splenic aspirates from patients with visceral Leishmaniasis *. Am. J. Trop. Med. Hyg. 32, 475-479. https://doi.org/10.4269/ajtmh.1983.32.475 (1983).

39. Schultz, G. et al. Consensus guidelines for the identification and treatment of biofilms in chronic nonhealing wounds. Wound Repair Regener. 25, 744-757. https://doi.org/10.1111/wrr.12590 (2017). 
40. Wilkinson, H. N., Mcbain, A. J., Stephenson, C. \& Hardman, M. J. Comparing the effectiveness of polymer debriding devices using a porcine wound biofilm model. Adv. Wound Care 5, 475-485. https://doi.org/10.1089/wound.2015.0683 (2016).

41. LABORATORY MANUAL IN MICROBIOLOGY - SECOND EDITION (2011). Sri Lanka College of Microbiologists Available at: http://slmicrobiology.lk/laboratory-manual-in-microbiology-second-edition-2011. (accessed 27 October 2021).

42. DNeasy PowerSoil Kit Handbook - QIAGEN. Available at: https://www.qiagen.com/us/resources/download.aspx?id=5a0517a7711d-4085-8a28-2bb25fab828a\&lang=en. (accessed 6 July 2021).

43. Castelino, M. et al. Optimisation of methods for bacterial skin microbiome investigation: Primer selection and comparison of the 454 versus MiSeq platform. BMC Microbiol. https://doi.org/10.1186/s12866-017-0927-4 (2017).

44. Chiodini, R. J. et al. Microbial population differentials between mucosal and submucosal intestinal tissues in advanced Crohns disease of the Ileum. PLoS ONE https://doi.org/10.1371/journal.pone.0134382 (2015).

45. 16S Metagenomic Sequencing Library Preparation Available at: https://support.illumina.com/downloads/16s_metagenomic_seque ncing_library_preparation.html. (accessed 6 July 2021).

46. Azeredo, J. et al. Critical review on biofilm methods. Crit. Rev. Microbiol. 43, 313-351. https://doi.org/10.1080/1040841x.2016. 1208146 (2016).

47. Fluorescence in situ hybridization (FISH) protocol for ... Available at: http://www.cancergeneticsitalia.com/wp-content/uploads/ 2010/04/CGI_FISH-Protocol_Paraffin_RUO.pdf. (accessed 6 July 2021).

48. Fiji. ImageJ Wiki Available at: https://imagej.net/Fiji. (accessed 6 July 2021).

49. cutadapt. PyPI Available at: https://pypi.org/project/cutadapt/1.2.1/. (accessed 6 July 2021).

50. Najoshi. najoshi/sickle. GitHub Available at: https://github.com/najoshi/sickle. (accessed 6 July 2021).

51. "Moving Pictures" tutorialg. QIIME 2 Docs Available at: https://docs.qiime2.org/2020.6/tutorials/moving-pictures/. (accessed 6 July 2021).

52. Person. Importing 13_8 Greengenes database for training classifier. QIIME 2 Forum (2019). Available at: https://forum.qiime2. org/t/importing-13-8-greengenes-database-for-training-classifier/12097. (accessed 6 July 2021).

53. Jbisanz. jbisanz/qiime2R: Import qiime2 artifacts to R. GitHub Available at: https://github.com/jbisanz/qiime2R. (accessed 21 October 2021).

54. McMurdie, P. J. \& Holmes, S. phyloseq: An R Package for Reproducible Interactive Analysis and Graphics of Microbiome Census Data. PLOS ONE Available at: http://dx.plos.org/https://doi.org/10.1371/journal.pone.0061217. (accessed 21 October 2021).

55. Wickham, H. et al. Welcome to the Tidyverse. J. Open Source Softw. 4, 1686 (2019).

56. Create Elegant Data Visualisations Using the Grammar of Graphics. Create Elegant Data Visualisations Using the Grammar of Graphics • ggplot2. Available at: https://ggplot2.tidyverse.org/. (accessed 21 October 2021).

57. Love, M. I., Huber, W. \& Anders, S. Moderated estimation of fold change and dispersion for RNA-seq data with DESeq2. Genome Biol. https://doi.org/10.1186/s13059-014-0550-8 (2014).

58. Siriwardana, H. V. Y. D., Senarath, U., Chandrawansa, P. H. \& Karunaweera, N. D. Use of a clinical tool for screening and diagnosis of cutaneous leishmaniasis in Sri Lanka. Pathog. Glob. Health 109, 174-183. https://doi.org/10.1179/2047773215y.0000000024 (2015).

59. NON-HEALING WOUNDS. Available at: https://www.facs.org/ /media/files/education/corecurriculum/nonhealing_wounds. ashx. (accessed 6 July 2021).

60. Parkash, O. Classification of leprosy into multibacillary and paucibacillary groups: An analysis. FEMS Immunol. Med. Microbiol. 55, 1-5. https://doi.org/10.1111/j.1574-695x.2008.00491.x (2009).

61. Bjarnsholt, T. et al. The in vivo biofilm. Trends Microbiol. 21, 466-474. https://doi.org/10.1016/j.tim.2013.06.002 (2013).

62. Ring, H. et al. Normal skin microbiota is altered in pre-clinical hidradenitis suppurativa. Acta Derm. Venereol. 97, 208-213. https:// doi.org/10.2340/00015555-2503 (2017).

\section{Acknowledgements}

University of Sri Jayewardenepura research grants (ASP/01/RE/MED/2018/54 and ASP/01/RE/MED/2019/40) for funding the project and National Centre for Primary Care \& Allergy Research, University of Sri Jayewardenepura, Sri Lanka for funding HbAlc tests.

\section{Author contributions}

T.D.J.K., P.C., A.J.M., M.M.W. wrote the main manuscript test. T.D.J.K. and P.C. prepared figures. All authors reviewed the manuscript.

\section{Competing interests}

The authors declare no competing interests.

\section{Additional information}

Supplementary Information The online version contains supplementary material available at https://doi.org/ 10.1038/s41598-021-02388-8.

Correspondence and requests for materials should be addressed to T.D.J.K.

Reprints and permissions information is available at www.nature.com/reprints.

Publisher's note Springer Nature remains neutral with regard to jurisdictional claims in published maps and institutional affiliations.

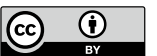

Open Access This article is licensed under a Creative Commons Attribution 4.0 International License, which permits use, sharing, adaptation, distribution and reproduction in any medium or format, as long as you give appropriate credit to the original author(s) and the source, provide a link to the Creative Commons licence, and indicate if changes were made. The images or other third party material in this article are included in the article's Creative Commons licence, unless indicated otherwise in a credit line to the material. If material is not included in the article's Creative Commons licence and your intended use is not permitted by statutory regulation or exceeds the permitted use, you will need to obtain permission directly from the copyright holder. To view a copy of this licence, visit http://creativecommons.org/licenses/by/4.0/.

(C) The Author(s) 2021 